\title{
Extensive use of peripheral angioplasty, particularly infrapopliteal, in the treatment of ischaemic diabetic foot ulcers: clinical results of a multicentric study of 221 consecutive diabetic subjects
}

\author{
E. FAgLiA ${ }^{1}$, M. MANTERO ${ }^{1}$, M. CAMINITI $^{1}$, C. CARAVAGGI $^{2}$, R. DE GIGLIO ${ }^{2}$, \\ C. PRITELLI ${ }^{2}$, G. CLERICI ${ }^{3}$, P. FRATINO $^{3}$, P. DE CATA $^{3}$, L. DALLA PAOLA ${ }^{4}$, G. MARIANI ${ }^{5}$, \\ M. POLI ${ }^{5}$, P. G. SETTEMBRINI ${ }^{5}$, L. SCIANGULA ${ }^{6}$, A. MORABITO ${ }^{7}$ \& L. GRAZIANI ${ }^{8}$ \\ From the ${ }^{1}$ Internal Medicine Unit, Diabetology Centre, Policlinico Multimedica, Sesto San Giovanni, Milano, ${ }^{2}$ Centre for the Study and \\ Treatment of Diabetic Foot Pathology, Abbiategrasso Hospital, Milano, ${ }^{3}$ Internal Medicine Unit, IRCCS Salvatore Maugeri Foundation, Pavia, \\ ${ }^{4}$ Endocrinology and Metabolism Unit, Diabetic Foot Centre, Villa Berica Hospital, Vicenza Italy, ${ }^{5}$ Diabetology Centre and Vascular Surgery Division, \\ S. Carlo Borromeo Hospital, Milano, ${ }^{6}$ Diabetology Centre, 'Felice Villa' Hospital, Mariano Comense, Como, ${ }^{7}$ Departement of Medicine, Surgery \\ and Dentistry, S. Paolo Hospital, University of Milan, Milan, ${ }^{8}$ Cardiovascular Catheterization Laboratory, 'Città di Brescia' Hospital, Brescia, Italy
}

\begin{abstract}
Faglia E, Mantero M, Caminiti M, Caravaggi C, De Giglio R, Pritelli C, Clerici G, Fratino P, De Cata P, Paola LD, Mariani G, Poli M, Settembrini PG, Sciangula L, Morabito A, Graziani L (Internal Medicine Unit, Diabetology Centre, Policlinico Multimedica, Sesto San Giovanni, Milano; Centre for the Study and Treatment of Diabetic Foot Pathology, Abbiategrasso Hospital, Milano; Internal Medicine Unit, IRCCS Salvatore Maugeri Foundation, Pavia; Endocrinology and Metabolism Unit, Diabetic Foot Centre, Villa Berica Hospital, Vicenza Italy; Diabetology Centre and Vascular Surgery Division, S. Carlo Borromeo Hospital, Milano; Diabetology Centre, 'Felice Villa' Hospital, Mariano Comense Como; Department of Medicine, Surgery and Dentistry, S. Paolo Hospital, University of Milan, Cardiovascular Catheterization Laboratory, 'Città di Brescia' Hospital, Brescia, Italy). Extensive use of peripheral angioplasty, particularly infrapopliteal, in the treatment of ischaemic diabetic foot ulcers: clinical results of a multicentric study of 221 consecutive diabetic subjects. J Intern Med 2002; 252: 225-232.
\end{abstract}

Objectives. To evaluate the feasibility, technical effectiveness and limb salvage potential of percutaneous transluminal angioplasty (PTA), particularly infrapopliteal, in diabetic subjects with ischaemic foot ulcer.

Design. Intervention study with PTA in consecutive series.
Setting. Six Diabetology Foot Centres and one Cardiovascular Catheterization Laboratory in Italy.

Subjects. Two hundred and twenty-one consecutive diabetic subjects hospitalized for ischaemic foot ulcer. Interventions. Peripheral arterial occlusive disease (PAOD) was investigated by means of foot pulses assessment, ankle-brachial-index (ABI), transcutaneous oxygen tension $\left(\mathrm{TcPO}_{2}\right)$ and duplex scanning. If non-invasive parameters suggested PAOD, angiography was performed and a PTA was carried out during the same session.

Main outcome measures. PTA feasibility, improvement of $\mathrm{ABI}$ and $\mathrm{TcPO}_{2}$, limb salvage rate, clinical recurrence.

Results. On angiography, two patients had stenoses which were $<50 \%$ of the vessel diameter. PTA was performed in 191 (85.3\%) of the 219 subjects with stenoses $>50 \%$, even when longer than $10 \mathrm{~cm}$ and/ or multiple/calcified. In 11 patients (5.8\%) PTA was performed in the proximal axis exclusively, in 81 $(42.4 \%)$ patients in the infrapopliteal axis exclusively and in $99(51.8 \%)$ in both the femoropopliteal and infrapopliteal axis. Both $\mathrm{ABI}$ and $\mathrm{TcPO}_{2}$ improved significantly after PTA $(P<0.0001)$. Clinical recurrence occurred in 14 subjects: 10 of whom underwent a second successful PTA. Of the 191 patients who underwent PTA, 10 (5.2\%) underwent an above-the-ankle amputation.

Conclusions. PTA, including infrapopliteal, is feasible in most diabetic subjects with ischaemic foot 
ulcer and is effective for foot revascularization. Clinical recurrence was infrequent and the procedure could successfully be repeated in most cases. In subjects treated successfully with PTA the abovethe-ankle amputation rate was low. PTA should be considered as the revascularization treatment of first choice in all diabetic subjects with foot ulcer and PAOD.

Keywords: diabetic foot ulcer, infrapopliteal angioplasty, major amputation, minor amputation, peripheral arterial occlusive disease.

\section{Introduction}

In diabetic subjects with ischaemic foot ulcer, successful revascularization reduces the rate of major amputation [1]. Whilst the effectiveness of peripheral by-pass, including distal by-pass, has been established, the role of percutaneous transluminal angioplasty (PTA) and especially the feasibility and effectiveness of infrapopliteal angioplasty remains to be elucidated [2]. Some reports indicate that the effectiveness and outcome of limb salvage is less favourable in diabetic compared with nondiabetic subjects [3], whilst others report no differences in outcome [4]. Recent reviews on diabetic foot care suggest that PTA should only be used for proximal arteries [5] and is seldom considered feasible [6].

Since July 1998, six diabetic foot centres employed non-invasive tests to assess peripheral arterial occlusive disease (PAOD) in all diabetic patients hospitalized for foot ulcer. All cases with non-invasive parameters suggestive of PAOD were referred to a specialized centre to be assessed angiographically and undergo PTA in the same session when possible.

We sought to evaluate PTA feasibility, especially infrapopliteal, foot revascularization effectiveness, complications, clinical recurrence and limb salvage rate in a population of consecutive diabetic patients hospitalized for ischaemic foot ulcer.

\section{Materials and methods}

Patient selection

All diabetic subjects hospitalized at our foot centres were evaluated for posterior tibial and dorsalis pedal pulses, ankle-brachial index ratio (ABI) by Doppler continuous wave technique, and transcutaneous oxygen tension $\left(\mathrm{TcPO}_{2}\right)$ tested on the dorsum of the foot. Duplex scanning was performed in cases with either reduced or absent foot pulses, $\mathrm{ABI}<1$ (in the absence of arterial calcification), or $\mathrm{TcPO}_{2}<50 \mathrm{mmHg}$ (in the absence of oedema). If at least two of these four exams were abnormal, arteriography was carried out and PTA revascularization performed concomitantly. In patients in which PTA was attempted unsuccessfully a by-pass operation was considered depending on the severity of obstructive disease and surgical risk factor. Our diagnostic and therapeutic protocol for ischaemic diabetic foot ulcer is show in Table 1.

\section{PTA procedure}

PTA was considered indicative for angiographically documented obstructions greater than $50 \%$ of the vessel lumen [7]. PTA revascularization technique was employed extensively: no stenosis or occlusion
1. Non-invasive evaluation of PAOD:

- Tibial posterior and pedal pulse examination

- Determination of Ankle-Brachial Index (ABI) measured with Doppler continuous wave technique

- Determination of transcutaneous oxygen tension $\left(\mathrm{TcPO}_{2}\right)$ measured at the dorsum of the foot If one pulse is absent or diminished or $\mathrm{TcPO}_{2}<50 \mathrm{mmHg}$ or $\mathrm{ABI}<1$

duplex scanning: if stenoses $>50 \%$ of veseel lumen

1. Angiography and concomitant PTA

2. PTA not feasible By-pass if possible

3. Infrapopliteal PTA after femoropopliteal by-pass if indicated

4. Surgical therapy if necessary
Table 1 Diagnostic and therapeutic protocol for diabetic foot ulcer 
was considered unsuitable a priori for PTA recanalization. Stenoses and occlusions greater than $10 \mathrm{~cm}$ in length, consecutive multiple stenoses, calcified occlusions, were treated by PTA. Vessel recanalization was considered complete when direct flow was obtained, with no significant residual stenosis along the artery (Fig. 1).

All procedures were performed under local anaesthesia through antegrade puncture of the ipsilateral common femoral artery. A 6-French vascular introducer was positioned to perform a preliminary angiographic study. An angiographic or percutaneous transluminal coronary angioplasty (PTCA) 0.014-0.035 in. guide wire was inserted to pass through any arterial obstructions and a 3.0 5.0 French balloon catheter, was used for the dilatation of $2-8 \mathrm{~mm}$ diameter arteries. The occurrence of thrombi was managed by catheter aspiration and/or urokinase infusion. In 12 cases self-expandable stents were inserted in $5-8 \mathrm{~mm}$ diameter vessels. Stents were not placed in the infrapopliteal arteries as the risk of thrombosis is very high in such low-flow vessels. During the procedure 3000-5000 IU of heparin was infused intra-arterially. If spasm occurred, $0.1-0.2 \mathrm{mg}$ of nitroglycerin was infused as an intra-arterial in bolus. All patients were prescribed ticlopidine $500 \mathrm{mg}$ per day for 30 days and acetyl salicylic acid $100 \mathrm{mg}$ per day indefinitely.

\section{Haemodynamic improvement}

Both the $\mathrm{ABI}$ and $\mathrm{TcPO}_{2}$ values were reassessed 3-6 days after the PTA procedure.

\section{Pain}

Pain changes after PTA were recorded.

\section{Recurrence}

Recurrence was assessed on the basis of pain reappearance, ulcer worsening or relapse. In these situations, $\mathrm{ABI}$ and $\mathrm{TcPO}_{2}$ were reassessed [8]. If those parameters were significantly worse $(<15 \%$ of the post-PTA value), a duplex scanning was performed and, in case of a positive test, the patient underwent another angiographic evaluation and, if possible, another PTA procedure.

\section{Complications}

Haematomas at the puncture site were not considered a significant side-effect unless requiring surgery or red cell transfusion. However, any event that prolonged hospital stay during PTA or required specific medical or surgical treatment immediately after PTA were recorded as a major complication.

Limb salvage

We considered limb salvage successful when the plantar stand was maintained, even when achieved by tarsal-metatarsal amputation [9]. Any above-theankle amputation was considered a failure.

\section{Clinical events during follow-up}

Patients were examined weekly after discharge and until ulcer healing, and were examined every 2 months thereafter. All subjects were provided with extra-deep rocker shoes with soft thermoformable leather and customized insoles.

Clinical recurrence, new ulceration, new major amputation or reamputation, and ischaemic pain recurrence were taken into account as events as well as the time and cause of death.

\section{Statistical methods}

For the consecutive series of patients considered, a descriptive statistics is reported as average values and standard deviation for the continuous variables and as percentages and contingency tables for the qualitative and discrete variables. $\mathrm{ABI}$ and $\mathrm{TcPO}_{2}$ mean values before and after PTA were compared by Student's $t$-test. The 5\% level has been assumed for testing the null hypothesis and 95\% level has been taken for constructing the confidence intervals.

\section{Results}

\section{Patient population}

Between July 1998 and July 2000, 402 consecutive diabetic subjects were hospitalized for foot ulcer, and in $221(55.0 \%)$ non-invasive vascular parameters suggesting PAOD were detected. These 221 patients were referred to a specialized centre for an angio- 

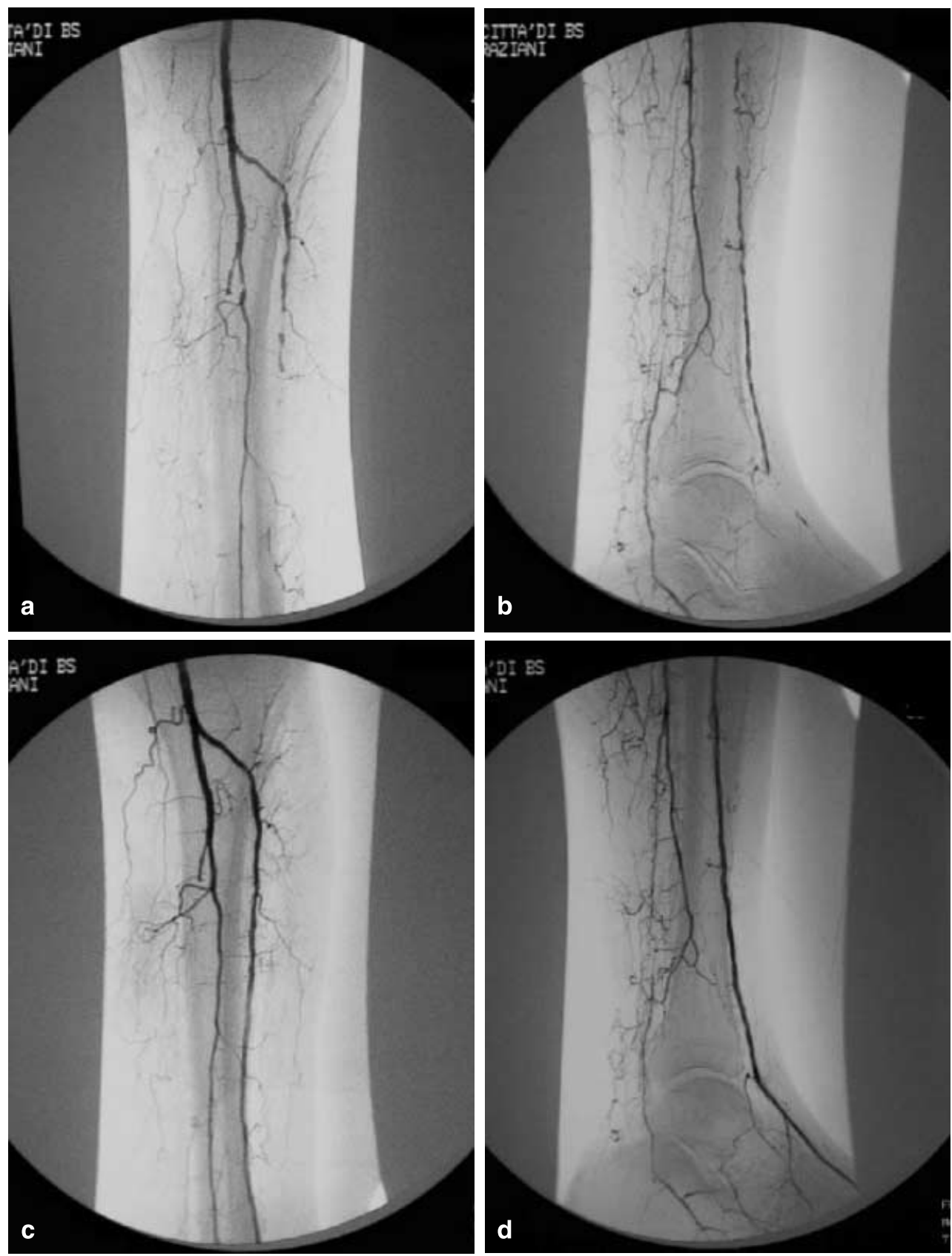

Fig. 1 (a, b) Occlusion of the posterior tibial artery, multiple obstructions with obstruction longer than $10 \mathrm{~cm}$ of the anterior tibial artery, occlusion of the pedal artery, critical stenosis of the peroneal artery. (c, d) Complete recanalization of peroneal and anterior tibial arteries, pedal artery included.

graphic study and, whenever possible, a concomitant PTA procedure. Two patients had no angiographic evidence of stenoses $>50 \%$ of vessel diameter: these two subjects were excluded from the analysis, thus leaving a study population of 219 individuals. Stenoses $>50 \%$ of vessel diameter were located exclusively in the iliac/femoro/popliteal axis in 11 patients, exclusively in the infrapopliteal axis in 81 patients, and in both femoro-popliteal and infrapopliteal axis in 127 patients. 


\section{PTA feasibility}

Percutaneous transluminal angioplasty was carried out in 191 subjects $(87.2 \%)$, in $28(12.8 \%)$ it was not feasible. The latter was because of complete calcified occlusion of the ostium and because of the inability to catheterize the proximal superficial femoral artery. Eleven $(5.8 \%)$ PTAs were performed exclusively in the proximal axis, $81(42.4 \%)$ exclusively in the infrapopliteal arteries (42.4\%), and 99 (51.8\%) in the femoropopliteal plus infrapopliteal arteries. Table 2 shows the number of PTA procedures performed in every artery of the lower limb with foot ulcer, the mean length of the stenoses and the occlusions recanalized, the percentage of the stenoses or occlusions $\geq 10 \mathrm{~cm}$ in length recanalized.

The demographic and clinical characteristics of the 191 subjects who underwent PTA are reported in Table 3.

\section{PTA technical effectiveness}

Transcutaneous oxygen tension and $\mathrm{ABI}$ were assessed both before and after PTA. $\mathrm{TcPO}_{2}$ was measured in 180 patients: in 11 cases $\mathrm{TcPO}_{2}$ data were lost. ABI was measured in 128 patients: in 62 patients it was impossible because of calcifications, and in one case the data were lost. $\mathrm{TcPO}_{2}$ increased from $21.3 \pm 12.6$ to $49.2 \pm 16.8 \mathrm{mmHg}$, and $\mathrm{ABI}$ from $0.53 \pm 0.15$ to $0.90 \pm 0.18$, both were highly significant $(P<0.0001)$. Figure 2 shows the values by class of $\mathrm{TcPO}_{2}$ and $\mathrm{ABI}$ before and after PTA.

\section{PTA complications}

No patients died during hospitalization for PTA. No puncture site haematoma required blood transfusion or surgery. A patient with ischaemic dilating cardiomyopathy had an acute renal failure which resolved within $48 \mathrm{~h}$ of the procedure and was managed medically. One subject complained of pain at the puncture site for approximately a month after PTA, with no documentable cause: abdominal sonography and peripheral arterial duplex scanning did not show any abnormality and pain resolved spontaneously.

\section{Recurrence}

Clinical recurrence occurred in 14 patients (7.3\%). The average time to clinical recurrence was 4.6 months, range 1-12 months. In 10 patients (71.4\%), a second PTA was performed successfully.

Pain

In all patients successfully treated with PTA the pain disappeared.

\section{Outcome of foot lesion}

Major amputation was carried out in 10 subjects $(5.2 \%)$, six above and four below the knee. Minor amputation was carried out in 83 patients: 20 tarsal-metatarsals, 35 of one or more rays, 28 of one or more toes. Ninety-eight patients healed with debridement and local dressing.

Out of the 28 subjects in whom PTA was not feasible, nine $(32.1 \%)$ underwent surgical revascularization. Of these nine subjects, one had a belowthe-knee amputation and one died in hospital because of acute myocardial infarction. Of the 19 subjects considered by the vascular surgeon not to be candidates for surgical reconstruction, six

Table 2 Recanalization with PTA performed in every artery of lower limb, mean length of the stenoses (50-99\% lumen diameter loss) and the occlusions ( $100 \%$ lumen loss) recanalized, percentage of stenoses and occlusions $\geq 10 \mathrm{~cm}$ of length recanalized

\begin{tabular}{|c|c|c|c|c|c|c|}
\hline \multirow[b]{2}{*}{ Artery } & \multicolumn{3}{|c|}{ Stenoses } & \multicolumn{3}{|c|}{ Occlusions } \\
\hline & $N$ & Mean length $(\mathrm{cm})$ & Length $\geq 10 \mathrm{~cm}(\mathrm{~N})$ & $N$ & Mean length $(\mathrm{cm})$ & Length $\geq 10 \mathrm{~cm}(N)$ \\
\hline Iliac trunk & 9 & $4.8 \pm 2.1$ & - & - & - & - \\
\hline Profunda femoral & 4 & $3.3 \pm 1.9$ & - & 3 & $3 \pm 1$ & - \\
\hline Common/superificial femoral & 58 & $8.7 \pm 6.4$ & $34.5 \%$ & 41 & $11.3 \pm 7.6$ & $51.2 \%$ \\
\hline Popliteal & 23 & $7.7 \pm 2.3$ & $17.4 \%$ & 16 & $6.4 \pm 3.4$ & $18.7 \%$ \\
\hline Anterior tibial/pedal & 33 & $6.3 \pm 4.4$ & $12.1 \%$ & 42 & $10.6 \pm 7.1$ & $38.1 \%$ \\
\hline Posterior tibial/plantar & 18 & $5.7 \pm 3.5$ & $16.7 \%$ & 17 & $11.5 \pm 7.8$ & $41.2 \%$ \\
\hline Peroneal & 42 & $7.6 \pm 4.6$ & $30.9 \%$ & 26 & $6.9 \pm 4.2$ & $15.4 \%$ \\
\hline
\end{tabular}


Table 3 Demographic and clinical characteristics of the study population $(N=191)$

\begin{tabular}{|c|c|}
\hline $\operatorname{Men}(N)$ & $120(62.8 \%)$ \\
\hline Women $(N)$ & $71(37.2 \%)$ \\
\hline Age (years) & $69.6 \pm 9.4$ \\
\hline Insulin therapy $(N)$ & $130(68.1 \%)$ \\
\hline Oral therapy $(N)$ & $61(31.9 \%)$ \\
\hline Diabetes duration (years) & $19.6 \pm 5.7$ \\
\hline Retinopathy (fundus oculi by ophthalmologist) $(N)$ & $148(77.5 \%)$ \\
\hline Vibration perception threshold measured by biothesiometer $>25 \mathrm{~V}(\mathrm{~N})$ & $98(60.9 \%)$ \\
\hline Insensitivity in $>5 / 9$ areas using Semmes-Weinstein monofilament $10 \mathrm{~g}(\mathrm{~N})$ & $96(60.8 \%)$ \\
\hline Achilles reflex absent $(N)$ & $97(50.8 \%)$ \\
\hline Renal impairment (creatinine value $>133 \mu \mathrm{mol} / \mathrm{L})(\mathrm{N})$ & $91(47.6 \%)$ \\
\hline Dialysis $(N)$ & $7(3.7 \%)$ \\
\hline Antihypertensive therapy $(N)$ & $151(79.1 \%)$ \\
\hline Coronary artery disease $(N)$ & $106(55.3 \%)$ \\
\hline Body mass index $\left(\mathrm{kg} \mathrm{m}^{-2}\right)$ & $26.4 \pm 3.4$ \\
\hline Smoking habit $(N)$ & $45(23.6 \%)$ \\
\hline \multicolumn{2}{|l|}{ Wagner grade $(N)$} \\
\hline 1 & $37(19.4 \%)$ \\
\hline 2 & $47(24.6 \%)$ \\
\hline 3 & $33(17.3 \%)$ \\
\hline 4 & $73(38.3 \%)$ \\
\hline 5 & $1(0.5 \%)$ \\
\hline Pain $(N)$ & $126(66.0 \%)$ \\
\hline Infection (positive culture exam) $(N)$ & $155(81.2 \%)$ \\
\hline Arterial media calcifications (X-ray of the feet) $(N)$ & $81(42.4 \%)$ \\
\hline Tibial posterior and/or dorsalis pulse absent $(N)$ & $184(96.3)$ \\
\hline Ankle-Brachial Index measured with Doppler continuous wave technique $(N=128)$ & $0.53 \pm 0.15$ \\
\hline Transcutaneous oxygen tension measured at the dorsum of the foot $(\mathrm{mmHg})(\mathrm{N}=180)$ & $21.3 \pm 12.6$ \\
\hline
\end{tabular}

Data are $N(\%)$ or mean \pm 1 SD.

(31.6\%) underwent a major amputation: five above and one below the knee.

\section{Follow-up}

One hundred and ninety subjects were followed until 31 December 2000. Follow-up information was not available for one patient after PTA and ulcer healing. The mean follow-up was $14.1 \pm 7.1$ months, range 5-30 months, median 12 months. In 15 (7.9\%) patients a new ulceration occurred: in nine at the original site and in six in another location. Ischaemic pain recurred in three subjects with worsening of non-invasive vascular parameters. These patients underwent a new arteriography that identified obstructive lesions at different sites from those originally treated. In these three patients a second PTA was successfully performed. In the remaining 12 subjects non-invasive vascular parameters did not vary and angiography was not deemed necessary. All the ulcers healed with medical dressings. No reamputation or new major amputations were performed. Ten $(5.3 \%)$ subjects died: seven due to ischaemic cardiopathy, one due to stroke, one due to pancreatic cancer, one due to geromarasmus. The average time interval between PTA and death was 12 months.

\section{Discussion}

In our population PTA was used extensively. We consider the term 'extensive PTA' to comprehend the technical aim and the clinical method.

The technical aim is to achieve the maximum available revascularization down to the foot independently of the severity and diffusion of the obstructive lesions. The majority of studies on PTA in infrapopliteal vessels report the treatment of isolated and short lesions, which are traditionally considered the only successful application of percutaneous recanalization [10]. In our experience PTA was even performed on very long stenoses or occlusions, multiple lesions on the same vessel and on calcified stenoses or occlusions.

Our clinical method considers every diabetic subject with foot ulcer and stenosis $>50 \%$ of vessel 

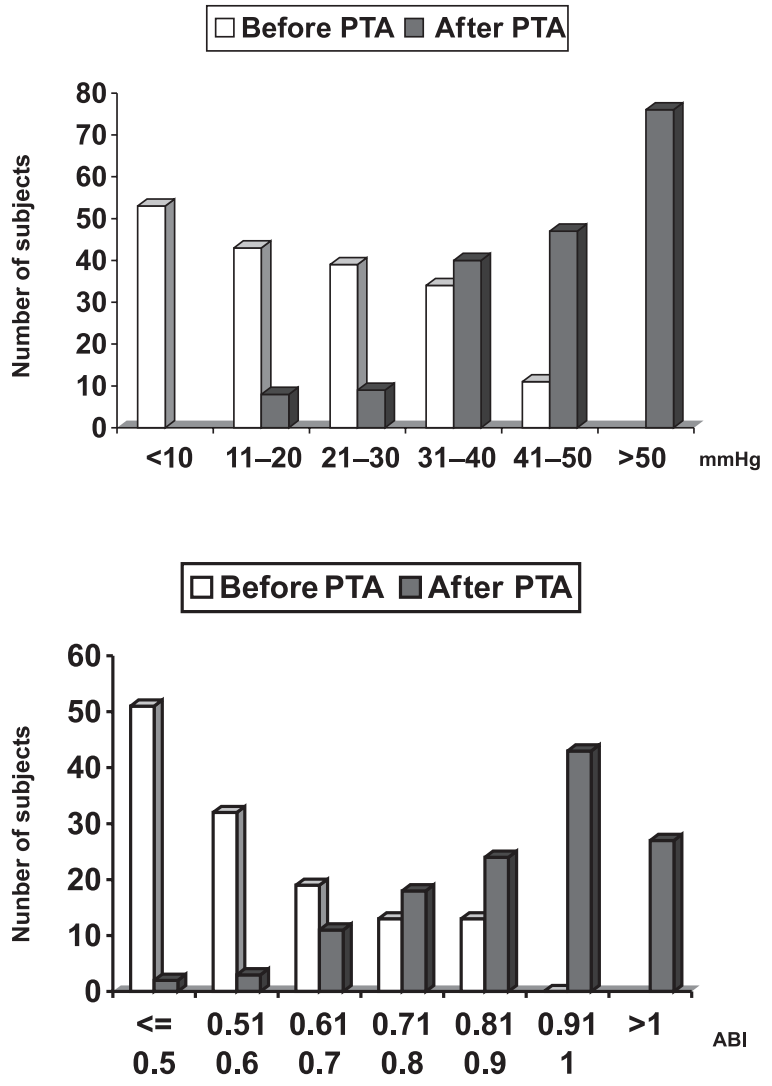

Fig. $2 \mathrm{TcPO}_{2}(n=180)$ and $\mathrm{ABI}(n=128)$ values by class before and after PTA procedure $(n=191)$.

diameter in the peripheral arterial tree a candidate for PTA revascularization, given the efficacy and low risk of this procedure. In fact, general or spinal anaesthesia is not necessary, there are no surgical wounds, the side-effects are minimal and mortality is very low $[11,12]$. A failed PTA does not preclude a subsequent by-pass operation.

For all these reasons we are currently considering PTA as the procedure of first choice in diabetic subjects with ischaemic foot ulcer, and we do not limit the indication for PTA to those patients who cannot undergo an operation [13]. Surgery is considered a second-line procedure after PTA failure [14].

The outcome of the foot lesion is the most relevant issue when assessing the efficacy of any therapeutic approach for ischaemic foot ulcer. Some authors report very good clinical results of PTA in claudicants, whilst in subjects with foot ulcer these are less encouraging $[15,16]$. As we have an efficient and low-risk procedure in PTA, we do not consider it correct, for ethical reasons, to randomize our patients for surgical revascularization.

We believe that the above-the-ankle amputation rate in the subjects who successfully underwent PTA is very low in comparison to literature data [17] and with our own historical data from the 1990s [18]. We believe that PTA revascularization has increased the likelihood of successfully performing minor amputations, which we consider as being limbsalvage procedures in subjects with large loss of tissue or gangrene who would otherwise most likely have undergone a major amputation [19].

Our current strategy is to perform PTA procedures in the same session as the angiographic evaluation. This approach reduces both patient stress and the amount of contrast administered, which are particularly relevant in subjects with cardiovascular diseases or nephropathy.

Percutaneous transluminal angioplasty is thought by some to be of limited efficacy because of its supposedly high recurrence rate [7]. In our experience, however, the clinical recurrence rate has been low. Especially, the recurrence was manageable by a second PTA in many patients. The morphological recurrence rate in our cases is probably higher than the clinical recurrence rate. Nevertheless, when tissue healing has occurred and no pain is present, we consider a possible delayed closure of the recanalysed vessel as clinically irrelevant. This is similar to continued limb salvage which occurs after closure of the surgical graft [20].

Our follow-up data of reamputation, new amputation, reulceration are encouraging. We would like to emphasize the stress that our foot centres place on counselling patients on foot care, correct use of special shoes and insoles [21].

Our data demonstrates that PTA, including infrapopliteal, is feasible in most diabetic subjects with PAOD and foot ulcer and is effective for foot revascularization. It is also feasible when very long, multiple or calcified stenoses or occlusions are present. Complications are rare if PTA is performed by a skilled physician, and PTA can be performed without any particular contraindications in patients with cardiovascular disease, nephropathy or at high surgical risk. Clinical recurrence rate is low and PTA can be successfully repeated in most cases. In this series, few patients required above-the-ankle amputation. All diabetic subjects with foot ulcer and 
PAOD should be considered as potential candidates for revascularization with PTA.

Our experience is available 'on line' at the web site: www.extrem-es-angioplasty.it.

\section{References}

1 LoGerfo FW, Gibbons GW, Pomposelli FB Jr et al. Trends in the care of the diabetic foot. Expanded role of arterial reconstruction. Arch Surg 1992; 127: 617-20.

2 Fraser SC, al-Kutoubi MA, Wolfe JH. Percutaneous transluminal angioplasty of the infrapopliteal vessels: the evidence. Radiology 1996; 200: 33-6.

3 Melliere D, Berrahal D, Desgranges P et al. Influence of diabetes on revascularisation procedures of the aorta and lower limb arteries: early results. Eur J Vasc Endovasc Surg 1999; 17: $438-41$.

4 Spence LD, Hartnell GG, Reinking G, Gibbons G, Pomposelli F, Clouse ME. Diabetic versus nondiabetic limb-threatening ischemia: outcome of percutaneous iliac intervention. Am J Roentgenol 1999; 172: 1335-41.

5 Caputo GM, Cavanagh PR, Ulbrecht JS, Gibbons GW, Karchmer AW. Assessment and management of foot disease in patients with diabetes. N Engl J Med 1994; 29: 854-60.

6 Levin ME. Preventing amputation in the patient with diabetes. Diabetes Care 1995; 18: 1383-94.

7 Pentecost MJ, Criqui MH, Dorros G et al. Guidelines for peripheral percutaneous transluminal angioplasty of the abdominal aorta and lower extremity vessels. A statement for health professionals from a special writing group of the Councils on Cardiovascular Radiology, Arteriosclerosis, Cardio-Thoracic and Vascular Surgery, Clinical Cardiology, and Epidemiology and Prevention, the American Heart Association. Circulation 1994; 89: 511-31.

8 Grollman J, Levin DC, Bettmann MA et al. Recurrent symptoms following lower extremity angioplasty: claudication and threatened limb. American College of Radiology. ACR Appropriateness Criteria. Radiology 2000; 215 (Suppl.): 95-9.

9 Garbalosa JC, Cavanagh PR, Wu G et al. Foot function in diabetic patients after partial amputation. Foot Ankle Int 1996; 17: 43-8.

10 Ouriel K. Peripheral arterial disease. Lancet 2001; 358: $1257-$ 64.

11 Kumpe DA, Rutherford RB. Percutaneous transluminal angioplasty for lower extremity ischemia. In: Rutheford RB, ed.
Vascular Surgery, 3rd edn. Philadelphia PA: WB Saunders, 1992; 759-61.

12 London NJ, Varty K, Sayers RD, Thompson MM, Bell PR, Bolia A. Percutaneous transluminal angioplasty for lower-limb critical ischemia. Br J Surg 1995; 82: 1232-5.

13 Rhodes JM, Gloviczki P, Bower TC, Panneton JM, Canton LG, Toomey BJ. The benefits of secondary interventions in patients with failing or failed pedal bypass grafts. Am J Surg 1999; 178: 151-5.

14 Gschwandtner MF, Minar F, Ahmadi A et al. Impact of different therapeutic alternatives in treatment of severe limb ischemia: experiences on 190 consecutive patients at a department of medical angiology. Vasa, 1999; 28: 271-8.

15 Melki JP, Fermand M, Riche MC, Lazareth I, Priollet P, Cormier JM. Treatment of diabetic arteriopathy. Importance of transluminal angioplasty. J Mal Vasc 1993; 18: 37-41.

16 Soder HK, Manninen HI, Jaakkola P et al. Prospective trial of infrapopliteal artery balloon angioplasty for critical limb ischemia: angiographic and clinical results. J Vasc Interv Radiol 2000; 11: 1021-31.

17 Reiber GE. The epidemiology of diabetic foot problems. Diabet Med 1996; 13 (Suppl. 1): S6-S11.

18 Faglia E, Favales F, Aldeghi A et al. Change in major amputation rate in a center dedicated to diabetic foot care during the 1980s: prognostic determinants for major amputation. J Diabetes Complications 1998; 12: 96-102.

19 Brodsky JW. Amputations on the foot and ankle. In: Mann RA, Coughlin MJ, eds. Surgery of the Foot and Ankle, 6th edn. St Louis: Mosby Year Book, 1993; 959-90.

20 Wagner HJ, Rager G. Infrapopliteal angioplasty: a forgotten region? Rofo Fortschr Geb Rontgenstr Neuen Bildgeb Verfahr 1998; 168: 415-20.

21 Faglia E, Favales F, Morabito A. New ulceration, new major amputation, and survival rates in diabetic subjects hospitalized for foot ulceration from 1990 to 1993: a 6.5-year follow-up. Diabetes Care 2001; 24: 78-83.

Received 6 December 2001; revision received 18 March 2002; accepted 29 May 2002.

Correspondence: Ezio Faglia, Internal Medicine Unit, Diabetology Center, Policlinico MultiMedica, Via Milanese 300, Sesto San Giovanni, Milano, Italy (fax: +39 022420 9463; e-mail: ezio. faglia@multimedica.it). 